\title{
Side-Chain and Main-Chain Conformational Transitions of Poly(L-1- and 2-naphthylalanine)s
}

\author{
Syun EgUSA, Masahiko SISIDO, ${ }^{*}$ and Yukio IMANISHI \\ Department of Polymer Chemistry, Kyoto University, Kyoto 606, Japan \\ * Research Center for Medical Polymers and Biomaterials, \\ Kyoto University, Kyoto 606, Japan
}

(Received December 3, 1985)

\begin{abstract}
Solvent and temperature-induced conformational transitions of poly(L-1- and 2naphthylalanine)s were investigated by circular dichroism in a 1,2-dichloroethane (DCE)/ trifluoroacetic acid (TFA) mixed solvent. The two polypeptides were in helical conformation in DCE in which the side chain chromophores are also arranged helically. Poly(L-1-naphthylalanine) showed a two-stage conformational transition upon the addition of TFA to the polymer solution in DCE. An intermediate conformation, which appeared at a TFA content between 0.5 and 3.0 $(\mathrm{v} / \mathrm{v}) \%$, was tentatively assigned to another helical conformation in which the orientation of the side-chain chromophores is different from that of the helical conformation in DCE. The final state, which appeared at higher TFA content than $3 \%$, was assigned to an extended $\beta$-structure. Poly(L-2naphthylalanine) showed a single conformational transition at a TFA content of $2-3 \%$. The final state was assigned to a randomly-coiled conformation. A temperature-induced conformational transition was observed for poly(L-2-naphthylalanine) in DCE/TFA mixed solvent with a TFA content of $1-5 \%$. In the mixed solvent, the polymer was in a randomly-coiled state at low temperatures and in a helical conformation at high temperatures.

KEY WORDS Poly(L-1-naphthylalanine) / Poly(L-2-naphthylalanine) / Conformation / Helix-Coil Transition / Circular Dichroism /
\end{abstract}

Conformations of novel aromatic polyamino acids, poly(L-1-naphthylalanine, poly(1-napAla) (I), and poly(L-2-naphthylalanine), poly(2-napAla) (II), have been investigated in
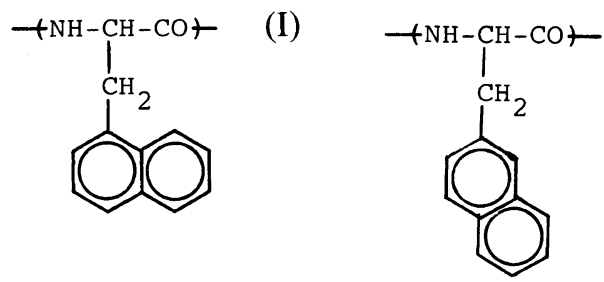

(II)

trimethyl phosphate (TMP). ${ }^{1,2}$ These polymers showed a strong exciton couplet at the ${ }^{1} \mathbf{B}_{\mathrm{b}}$ absorption band of the naphthyl group, suggesting that the naphthyl groups are helically arranged along a helical main chain. The helical arrangement of side chains has been rarely observed in other aromatic polypeptides, such as poly(arylmethyl L-aspartate) $\mathrm{s}^{3,4}$ and poly(arylmethyl L-glutamate) $\mathrm{s}^{5}$ in solution. In the latter polymers, the spacer group which connects the aromatic group to the main chain is so long that the aromatic group may rotate almost freely in solution, even when their main chains are in helical conformations. In the case of poly(arylalanine)s, the aromatic groups are linked to the main chain by a single methylene group and the side-chain rotations are severely restricted, resulting in the helical arrangement of the side chain.

Since the helical arrangement of the side chains are observed in poly(1- and 2-napAla)s, it should be of interest to examine the stability and the unfolding processes of the main-chain and the side-chain helical structures in solu- 
tion. ${ }^{6}$ In this report, solvent and temperatureinduced conformational transitions of poly(1and 2-napAla)s in 1,2-dichloroethane (DCE)/ trifluoroacetic acid (TFA) mixed solvent are described. The conformational transition was studied by circular dichroism (CD) spectroscopy. For comparison, the CD spectra of linear dipeptides of 1- and 2-napAla were measured in the same mixed solvent.

\section{EXPERIMENTAL}

\section{Materials}

Poly(L-1- and 2-naphthylalanine)s were prepared by the same procedure as described previously. ${ }^{1,2}$ Since homopolymers of 1- and 2napAla were insoluble in any organic solvents, A-B-type block copolymers of poly(1- and 2napAla)s with poly $(\gamma$-benzyl DL-glutamate) (number average degree of polymerization = 150) were used as before. Since the polyglutamate unit does not affect CD spectra and optical rotation of polynaphthylalanines, the block copolymers are referred to simply as poly(1- and 2-napAla)s, hereafter. The degree of polymerization of the poly(1- or 2-napAla) unit, which was estimated from the polymerization conditions, is approximately equal to or larger than 40 . The block copolymers were prepared in DCE solution and after completion of the polymerization, the polymer solution was diluted with DCE to an appropriate concentration for spectroscopic measurements.

Preparations of linear dipeptides of 1- and 2napAla, e.g., $N$-acetyl-L-1(2)-naphthylalanylL-1(2)-naphthylalanine methyl ester [Ac(1(2)napAla $)_{2} \mathrm{OMe}$ ], will be reported elsewhere. ${ }^{7}$

\section{Measurements}

CD spectra and optical rotations were measured on a JASCO J-20 spectropolarimeter using quartz cells with an optical path length of $0.2 \mathrm{~mm}$ for $\mathrm{CD}$ and $10 \mathrm{~mm}$ for ORD. The temperature was controlled by circulating thermostated water through an outer cylinder of the cell over the temperature range of 10 $50^{\circ} \mathrm{C} .{ }^{13} \mathrm{C}$ NMR was recorded on a JEOL FX90Q instrument $(22.5 \mathrm{MHz})$ at $30^{\circ} \mathrm{C}$ in DCE- $d_{4}$. Since the signals from the solvent and from the polyglutamate unit were excessive, they were eliminated by a $180^{\circ}-\tau-90^{\circ}$ pulse sequence $(\tau=0.5 \mathrm{~s})$.

\section{RESULTS AND DISCUSSION}

$$
\text { Poly(L-1-naphthylalanine) }
$$

\section{Circular Dichroism and Optical Rotation in a Dichloroethane/Trifluoroacetic Acid Mixed Solvent}

Figure 1 shows the CD spectra of poly(1napAla) in a mixed solvent with varying amounts of TFA. A distinct exciton couplet is observed at the ${ }^{1} \mathbf{B}_{b}$ absorption band of naphthyl chromophore in the absence of TFA. The molar ellipticities, $[\theta]_{218}=-3.8 \times 10^{5},[\theta]_{233}=$ $6.0 \times 10^{5}\left(\mathrm{deg} \mathrm{cm}^{-2} \mathrm{dmol}^{-1}\right)$, are about the same as those observed in TMP. The marked exciton couplet suggests a helical arrangement of the naphthyl groups along a helical main chain. Even a trace amount of TFA $(0.5 \%)$ drastically changed the $C D$ spectrum, but a considerable exciton splitting was still present. On further addition of TFA, the spectrum gradually changed and eventually the exciton splitting disappeared.

An isodichroic point was seen at $215 \mathrm{~nm}$ throughout the change in solvent composition, except for the case of $0 \%$ TFA. The change in CD spectra suggests a two-stage conformational transition of poly(1-napAla) with the addition of TFA. The two-stage transition is more evident in Figure 2, in which molar ellipticities at 233 and $226 \mathrm{~nm}$ and molar optical rotation $[\mathrm{m}]$ at $400 \mathrm{~nm}$ are plotted as functions of TFA content. The molar ellipticities abruptly changed by the addition of $0.5 \%$ TFA, whereas the change in the optical rotation is small at this TFA concentration. Since the molar rotation at $400 \mathrm{~nm}$, which falls outside the absorption band of naphthyl group, may be governed more by the helical arrange- 
Poly(1- and 2-naphthylalanine)s

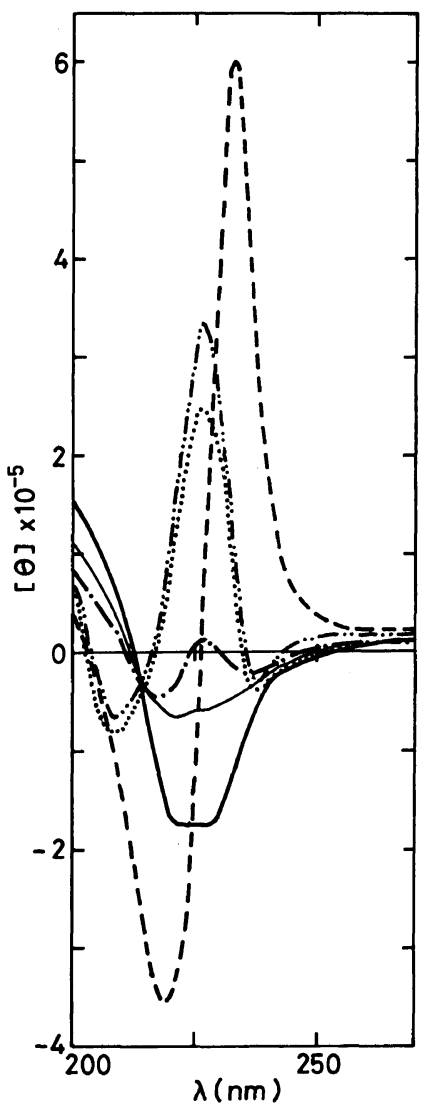

Figure 1. CD spectra of poly(1-napAla) in DCE/TFA mixed solvent with varying amounts of TFA at $20^{\circ} \mathrm{C}$. TFA content $(\mathrm{v} / \mathrm{v} \%)$ : $0(----), 0.5(---), 1.0(\cdots)$, $2.0(--)^{\circ}, 3.0(-)$, and $5.0(-)$.

ment of the main-chain amide chromophores than by the side-chain naphthyl chromophores, the insensitivity of $[\mathrm{m}]_{400}$ to the small amount of TFA suggests that the main-chain conformation does not change during the first transition.

Both $[\theta]_{226}$ and $[m]_{400}$ discontinuously changed within a narrow range of the TFA content of $1.5-3.0 \%$. This indicates that both main-chain and side-chain conformations change during the second transition. It should be noted that even after adding more than $10 \%$ TFA, the molar rotation continued to have a considerably large negative value, indicating that the final state also possesses some regular

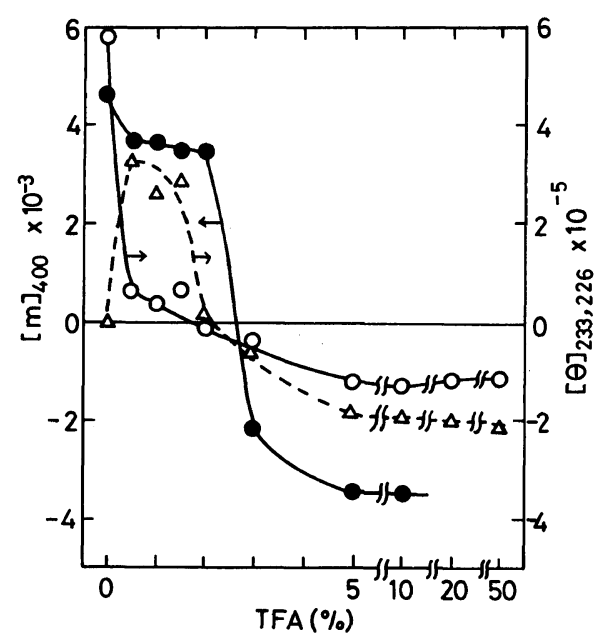

Figure 2. Effects of the addition of TFA on $C D$ and ORD spectra of poly(1-napAla) in DCE at $20^{\circ} \mathrm{C}$. $[\theta]_{226}$ $\left(--\triangle^{--}\right),[\theta]_{233}\left(-\mathrm{O}^{-}\right)$, and $[m]_{400}(-\bigcirc-)$.

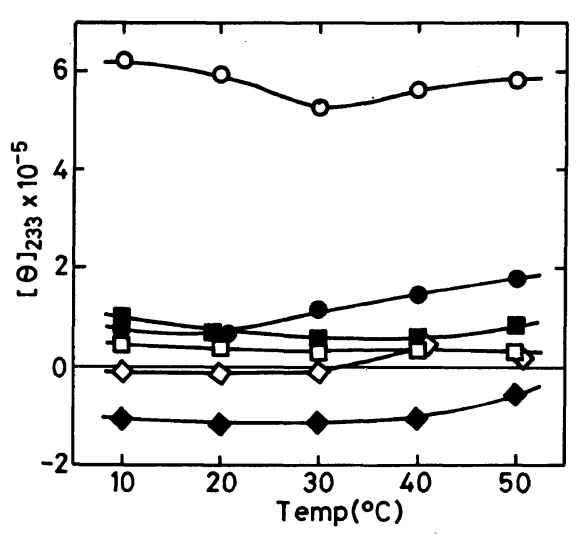

Figure 3. Temperature dependence of $[\theta]_{233}$ of poly(1-napAla) in DCE/TFA mixed solvent with varying amounts of TFA. TFA content $(\mathrm{v} / \mathrm{v} \%): 0(0), 0.5$ $(\bigcirc), 1.0(\square), 1.5(\square), 2.0(\diamond)$, and $5.0(\diamond)$.

main-chain conformation, which is assigned to a $\beta$-structure in the latter section.

The molar ellipticity was measured at TFA content of $0,0.5,1.0,1.5,2.0$, and $5.0 \%$ over a temperature range of $10-50^{\circ} \mathrm{C}$ (Figure 3). However, no significant temperature dependence was detected. The absence of temperature-induced conformational transition suggests that the carbonyl oxygen and nitrogen atoms of the amide bonds are intramolecularly hydrogen-bonded both in the intermediate and 


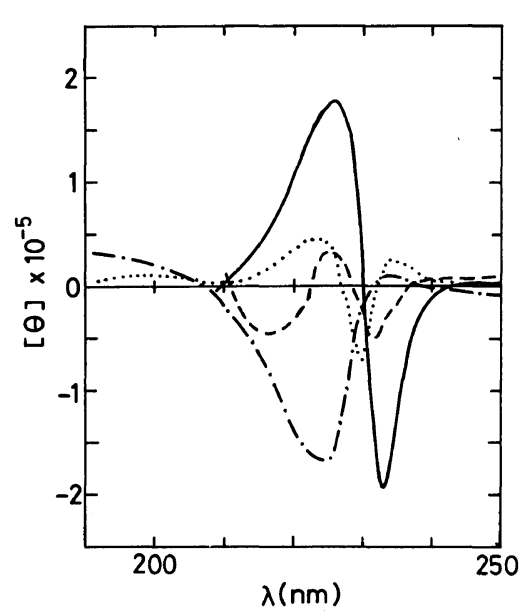

Figure 4. CD spectra of Ac-(1-napAla $)_{2}$-OMe in TMP $(--)$ ) and in DCE/TFA $(5.0 \%)(---)$, and Ac- $(2-$ napAla) $)_{2}$-OMe in TMP (-----) and in DCE/TFA $(5.0 \%)$ $(\longrightarrow) .25^{\circ} \mathrm{C}$.

in final states. ${ }^{8}$ A temperature-induced conformational transition would have been observed if the final state were a randomly-coiled conformation. This is actually the case for poly(2-napAla) as will be discussed later.

\section{Circular Dichroism of a Linear Dipeptide}

In order to assign the conformations observed in the DCE/TFA mixed solvents, the CD spectra of a linear dipeptide, Ac-(1-napAla $)_{2}$ OMe, was measured in a DCE/TFA $(5 \%)$ mixture and in TMP (Figure 4). The dipeptide shows quite different $C D$ patterns in the two solvents and the spectrum in TMP resembles that of poly(1-napAla) in a DCE/TFA mixture containing a large amount of TFA. Rizzo and Jäckle $^{9}$ reported that linear dipeptides of aromatic amino acids are in extended conformations in aprotic solvents and folded conformations in protic solvents. It is therefore likely that the final state of poly(1-napAla) which showed a similar CD pattern to that of the dipeptide in TMP (aprotic solvent), is an extended $\beta$-structure.

\section{Conformations of Poly(1-napAla)}

The CD data suggest that poly(1-napAla) in a DCE/TFA mixed solvent has a two-stage conformational transition. The initial state A, was assigned to a right-handed $\alpha$-helix carrying a helical array of the naphthyl groups along the main chain. ${ }^{10}$ The main chain of the intermediate state B may have the same helical structure as A, but it is unclear whether the side chains take on a helical arrangement. Previously ${ }^{1,10}$ we made empirical conformation energy calculations for helical poly(1napAla) and found that two kinds of sidechain arrangements are possible for the $\alpha$ helical main-chain backbone.* The rotational angles of the lower-energy conformation (form A) are: $\left(\phi, \psi, \chi_{1}, \chi_{2}\right)=\left(-67^{\circ},-40^{\circ}, 177^{\circ}\right.$, $\left.257^{\circ}\right)$. Those for the higher-energy conformation (form B) are: $\left(-54^{\circ},-50^{\circ}, 267^{\circ}, 111^{\circ}\right)$.

Theoretical CD spectra for the two conformations were calculated ${ }^{10}$ and are shown in Figure 5. It is interesting to note that the theoretical CD spectrum for form A resembles the CD spectrum in DCE (Figure 1) and the theoretical $\mathrm{CD}$ for form $\mathrm{B}$ resembles that of the intermediate state. Therefore, we tentatively assigned the intermediate conformation to the $\alpha$-helical conformation with the side-chain arrangement in form $B$.

The drastic change of $[\mathrm{m}]_{400}$ in Figure 2 indicates that the final state $\mathrm{C}$ had a different main-chain conformation from $\alpha$-helix. However, the large negative value of the molar rotation suggests some regularity in the final state. A comparison of the CD curve with that of the linear dipeptide [Ac(1-napAla $)_{2} \mathrm{OMe}$ ] suggested an extended $\beta$-structure for the main-chain conformation in the final state. The intermolecular association due to the formation of the $\beta$-structure is supported by the turbidity which appeared a few days after the addition of more than $5 \%$ TFA. This observation is in contrast to the case of poly(2-napAla)

* In ref 1 , the most probable conformation of poly(1-napAla) in TMP has been suggested to be a right-handed $\delta$ helix. However, recent calculations for a series of sequential polypeptides, poly[Lys $(Z)_{m}-1$-napAla] $(m=0,1,2,3$, and 4), indicated that the homopolymer of 1 -napAla takes on a right-handed $\alpha$-helix in TMP (ref 10). 


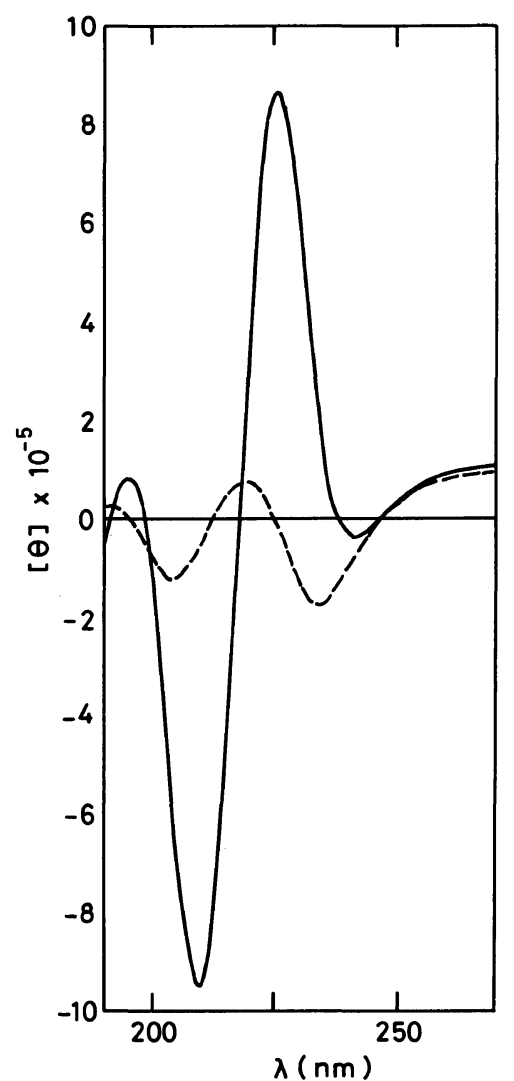

Figure 5. Theoretical CD spectra of poly(1-napAla) in right-handed $\alpha$-helical conformations. The side-chain conformation is in the A form (-), and B form (--- ).

in the same mixed solvent, where no turbidity was detected.

The above assignment is supported by the ${ }^{13} \mathrm{C}$ NMR data. No peak assignable to the naphthylmethyl group was detected in the NMR spectrum measured in the mixed solvent with varying amounts of TFA $(0-5 \%)$. The absence of NMR peak contrasts the observation of 2-naphthylmethyl peaks in poly(2napAla) in a random coil state (see below) and indicates that the mobility of the naphthyl group in poly(1-napAla) is highly restricted irrespective of the TFA concentration. The rigid structure is compatible with the two-stage transition: the $\alpha$-helix with a side-chain arrangement in the A form $\rightarrow \alpha$-helix with a side-

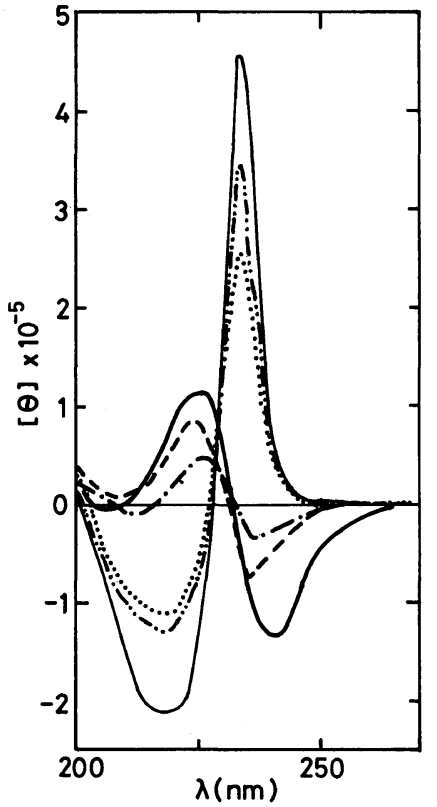

Figure 6. CD spectra of poly(2-napAla) in DCE/TFA mixed solvent with varying amounts of TFA at $20^{\circ} \mathrm{C}$. TFA content $(\mathrm{v} / \mathrm{v} \%): 0(-), 1.0(---), 2.0(\cdots)$, $3.0(--\square), 5.0(---)$, and $10.0(\square)$

chain arrangement in the $\mathrm{B}$ form $\rightarrow \beta$-structure.

Poly(2-naphthylalanine)

\section{Circular Dichorism and Optical Rotation}

Figure 6 shows the CD spectra of poly(2napAla) in a DCE/TFA mixed solvent with varying content of TFA. Poly(2-napAla) was found to take on a left-handed helix in TMP in which the side groups are also helically arranged. $^{2}$ The CD spectrum in pure DCE exhibits the same pattern as that in TMP. The molar ellipticities in DCE are: $[\theta]_{218}=-2.1 \times$ $10^{5},[\theta]_{233}=4.6 \times 10^{5}\left(\mathrm{deg} \mathrm{cm}^{-2} \mathrm{dmol}^{-1}\right)$. Unlike poly(1-napAla), the change of the $C D$ spectrum of poly(2-napAla) upon addition of TFA may be interpreted, as a first approximation, in terms of a single conformational transition, since an approximate isodichroic point is observed around $230 \mathrm{~nm}$.

The conformational transition is more clearly shown in Figure 7, where the molar 


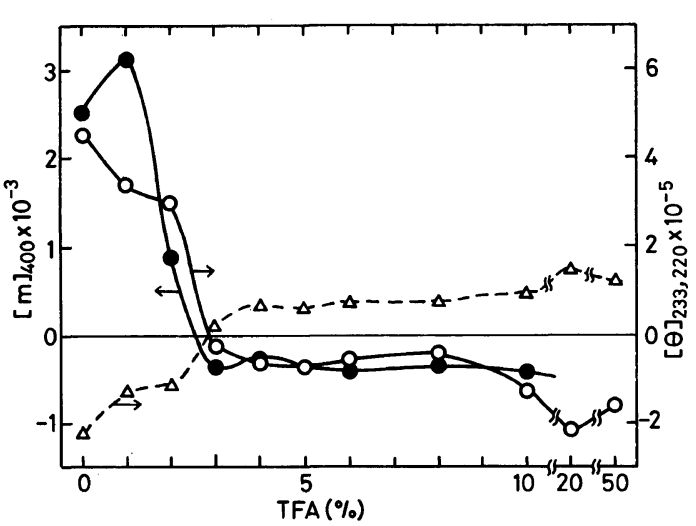

Figure 7. Effects of the addition of TFA on CD and ORD spectra of poly(2-napAla) in DCE at $20^{\circ} \mathrm{C}[\theta]_{220}$ $\left(--\triangle^{--}\right),[\theta]_{233}\left(-\mathrm{O}^{-}\right)$, and $[m]_{400}(-\bigcirc-)$.

ellipticities $[\theta]$ and the molar rotation $[m]$ are plotted against the TFA content. A sharp discontinuous change was observed at the TFA content of $2-3 \%$, and the further addition of TFA caused no appreciable change in the CD spectra and optical rotation. The final state observed in the presence of more than $3 \%$ of TFA, may be a randomly-coiled conformation because the CD intensities and the optical rotation are small. The randomly-coiled state is further supported by the similarity of the CD pattern of the final state in Figure 7 to that of the linear dipeptide of 2-napAla in a DCE/ TFA (5\%) mixture (Figure 4, solid curve). It should be noted that in the latter CD spectrum, the molar ellipticity was calculated on the basis of two naphthyl groups (one dipeptide molecule). Therefore, the CD spectrum of poly(2-napAla) in DCE/TFA $(5 \%)$ is similar to that of $\mathrm{Ac}(2 \text {-napAla })_{2} \mathrm{OMe}$ in the same solvent in shape and magnitude. This indicates that the CD of poly(2-napAla) is mainly determined by the naphthyl-naphthyl interaction in dipeptide units which take on a variety of conformations in a DCE/TFA $(5 \%)$ mixed solvent.

\section{Temperature-Induced Conformational Transi- tion}

Figure 8 shows $[\theta]_{233}$ as a function of tem-

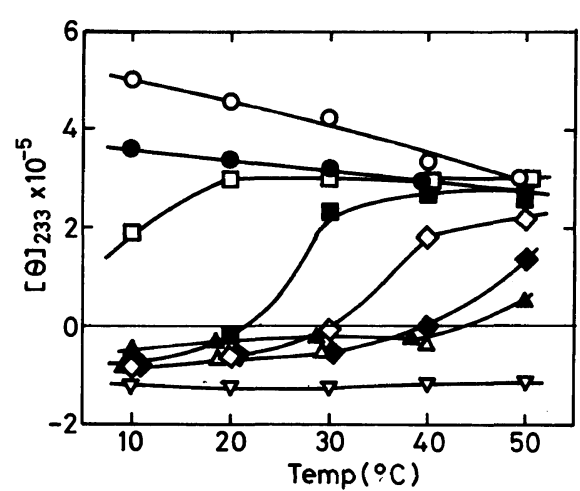

Figure 8. Temperature dependence of $[\theta]_{233}$ of poly(2-napAla) in DCE/TFA mixed solvent with varying amounts of TFA. TFA content $(\mathrm{v} / \mathrm{v} \%)$ : $0(0), 1.0$ $(\bigcirc), 2.0(\square), 3.0(\square), 4.0(\diamond), 5.0(\diamond), 6.0(\triangle), 8.0(\mathbf{\Delta})$, and $10.0(\nabla)$.

perature in a DCE/TFA mixture containing different amounts of TFA. A temperatureinduced helix(high temp.)-coil(low temp.) transition is evident from the figure. This inverse transition is reversible over this temperature range. The inverse helix-coil transition has been observed for several synthetic polypeptides in binary mixtures of helix-supporting solvent and helix-breaking acid (TFA and dichloroacetic acid). ${ }^{8}$ The observation of the inverse transition supports the above assignment of the final state to a randomlycoiled conformation. In the randomly-coiled conformation, the oxygen and nitrogen atoms of the main-chain amide bonds are hydrogenbonded to the strong acid and the hydrogen bonds are more stable at low temperatures, resulting in an inverse helix-coil transition. ${ }^{8}$ The observation of the thermal helix-coil transition in poly(2-napAla) is in contrast with the absence of any temperature effect in poly(1napAla) in Figure 3. This difference can be interpreted by the difference of the conformations of the final states, i.e., extended $\beta$-structure of poly(1-napAla) and randomly-coiled conformations of poly(2-napAla).

\section{Conformations of Poly(2-napAla)}

Unlike the case of poly(1-napAla), poly(2- 
napAla) showed a single helix-coil transition in DCE/TFA mixed solvents. The final state at high TFA concentration was assigned to a randomly-coiled conformation by means of CD and ORD spectroscopy and by the observation of thermal helix-coil transition. The randomly-coiled conformation is further supported by the appearance of naphthyl peaks in ${ }^{13} \mathrm{C}$ NMR in DCE/TFA $(10 \%)$ mixture at $30^{\circ} \mathrm{C}$. This is in contrast with the case of poly(1-napAla), in which no peak assignable to the naphthylmethyl group was detected. The NMR peak disappeared on increasing the temperature to $50^{\circ} \mathrm{C}$. The disappearance of the NMR signal, however, contradicts the result of CD spectroscopy (Figure 8), where no coil to helix transition was observed under the same conditions. A possible explanation for this discrepancy is that the chain motion may become sluggish before a regular arrangement of the naphthyl groups is attained.

The difference in the behavior of the two polymers in DCE/TFA mixture may be interpreted by differences in the affinities of the two polypeptide chains to TFA, caused not only by differences of the main-chain conformations of the two polypeptides [right-handed $\alpha$-helix for poly(1-napAla $)^{1}$ and left-handed helix for poly(2-napAla) ${ }^{2}$, but also by differences of the side-chair arrangements of the two polypeptides.

\section{REFERENCES}

1. M. Sisido, S. Egusa, and Y. Imanishi, J. Am. Chem. Soc., 105, 1041 (1983).

2. M. Sisido, S. Egusa, and Y. Imanishi, J. Am. Chem. Soc., 105, 4077 (1983).

3. M.-H. Loucheux-Lefebvre, C. Duflot, and G. Weill, Biopolymers, 14, 469 (1975).

4. M. Sisido, A. Okamoto, S. Egusa, and Y. Imanishi, Polym. J., 17, 1253 (1985).

5. P. Le Barny and M.-H. Loucheux-Lefebvre, Biopolymers, 12, 2831 (1973).

6. A. Teramoto and H. Fujita, Adv. Polym. Sci., 18, 65 (1975).

7. S. Egusa, M. Sisido, and Y. Imanishi, Bull. Chem. Soc. Jpn., in press.

8. Reference 6, p 87.

9. V. Rizzo and H. Jäckle, J. Am. Chem. Soc., 105, 4195 (1983).

10. M. Sisido and Y. Imanishi, Macromolecules, in press. 